\title{
ESSM 2011: Risikofaktoren, Begleiterkrankungen und Therapiemanagement der ED
}

— Die moderne Therapie der Erektilen Dysfunktion (ED) stand auf dem 14. Kongress der European Society for Sexual Medicine (ESSM) im Fokus. Im Rahmen eines Satellitensymposiums thematisierten die Referenten unter anderem die aktuellen Forschungsergebnisse auf dem Gebiet der ED und seiner Komorbiditäten: Demnach ist das häufige gemeinsame Auftreten der ED mit kardiovaskulären Erkrankungen, Diabetes und/oder BPS oft durch ähnliche Risikofaktoren begründet. Epidemiologische Studien wie die Kölner Studie [Braun, M. et al: Int J Impot Res 2000] unterstreichen die hohe Prävalenz von ED und Begleiterkrankungen. Männer mit ED haben demnach wesentlich häufiger Symp- tome des unteren Harntraktes $(72,2 \%$ vs. $37,7 \%)$, Hypertonie $(32,0 \%$ vs. $13,6 \%)$ und Diabetes (20,2\% vs. $3,2 \%$ ) als die Vergleichsgruppe ohne ED.

Generell empfahlen die Referenten bei der Anamnese von ED-Patienten stets auf Symptome weiterer Erkrankungen zu achten. FirstLine-Therapieempfehlung ist nach den EAULeitlinien neben der Lebensstilveränderung die medikamentöse Behandlung mit PDE 5-Hemmern [Hatzimouratidis, K. et al: Eur Urol2010;57(5):804-14] wie Tadalafil (Cialis ${ }^{\circledR}$ ). Dieser ist in Deutschland mit der Dosierung von $5 \mathrm{mg}$ der einzige für eine einmal tägliche Therapie zugelassene Wirkstoff. Aufgrund seiner langen Halbwertszeit von 17,5 Stun- den kann in der Regel innerhalb von fünf Tagen ein konstanter Wirkspiegel erreicht werden. Demnach ist die Wirkung bei Therapieerfolg und sexueller Stimulation jederzeit abrufbar. Die einmal tägliche Gabe von Tadalafil 5 mg gestattet ED-Patienten die Tabletteneinnahme zeitlich und psychologisch weitestgehend von der sexuellen Aktivität zu trennen. Die Referenten beim Satellitensymposium waren sich einig, dass unabhängig von Komorbiditäten die Bedürfnisse des Patienten ein wesentlicher Faktor bei der Wahl des PDE 5-Hemmers seien. Ein tägliches Präparat wie Tadalafil $5 \mathrm{mg}$ könne durch den möglichen Gewinn an zeitlicher Flexibilität und Spontaneität eine wirksame und verträgliche Alternative zur Bedarfstherapie darstellen.

Nach Informationen von Lilly, Bad Homburg

\section{mCRPC: Nebenwirkungen proaktiv begegnen}

— Cabazitaxel (Jevtana ${ }^{\oplus}$ ) ist seit einem Jahr für die Behandlung rezidivierter Patienten mit metastasiertem kastrationsresistentem Prostatakarzinom (mCRPC) nach DocetaxelVersagen freigegeben. In der Zulassungsstudie TROPIC reduzierte das Präparat das relative Sterberisiko der Patienten um $30 \%$ im Vergleich zur Behandlung mit Mitoxantron (HR 0,70; $p<0,0001$ ) [de Bono JS et al., Lancet 2010, 376: 1147-54]. Der Überlebensvorteil zeigte sich unter anderem unabhängig vom Alter der Behandelten, dem Vorliegen von tumorbedingten Schmerzen, der Progredienz des PSA-Wertes und der vorangegangenen kumulativen Docetaxel-Dosis. Die Sorge, dass das in der TROPIC-Studie beobachtete Neutropenierisiko den klinischen Einsatz von Cabazitaxel limitieren könnte, bestätigt sich im klinischen Alltag nicht, betonte Dr. Bernhard Heinrich, Augsburg. Alle elf Probanden, die Heinrich nach Docetaxel-Versagen noch einmal mit Cabazitaxel behandelte, profitierten davon: Bei den meisten Patienten bildete sich der Tumor noch

\section{Trospiumchlorid: neue Packungsgrößen}

— Seit Kurzem ist die Retardformulierung von Trospiumchlorid (uriVesc ${ }^{\oplus}$ ) zur Behandlung der überaktiven Blase in zwei neuen, erstattungsfähigen Packungsgrößen erhältlich: Die 30er-Packung ersetzt die 28erPackung, um die Therapie zu starten, und die 100er-Packung löst die 84er-Packung ab, um die Therapie langfristig fortzusetzen. Das Medikament hat außerdem unter den gängigsten Blasenspasmolytika die niedrigsten Tagestherapiekosten (30,29€ und $69,23 €$ ). Die Retardformulierung von Trospiumchlorid ist bei gutem Wirkprofil deutlich besser verträglich als die schnell freisetzende Form. In einer aktuellen nicht interventionellen Studie (NIS) der Firma SAM wurden die Daten der beiden Zulassungsstudien bestätigt. Ein weiterer Aspekt, der den Einsatz von Trospiumchlorid Retardkapseln unterstreicht: Die Patienten berichteten über eine hochsignifikante Verbesserung ihrer Lebensqualität unter uriVesc ${ }^{\circledast}$.

Nach Informationen von

Rottapharm|Madaus, Köln einmal zurück, was in der Regel mit einer Abnahme der tumorbedingten Beschwerden einherging. In den anderen Fällen kam es zu einem Wachstumsstopp, sodass das weitere Tumorwachstum und die dadurch bedingten Beschwerden hinausgezögert wurden.

Trotz der zum Teil intensiven Vorbehandlung haben alle Patienten die volle CabazitaxelDosis plus Prämedikation erhalten und diese auch gut vertragen. Das galt auch für die hämatologischen Nebenwirkungen, die sich zuverlässig handhaben beziehungsweise mit zusätzlicher G-CSF-Gabe (Granulozyten-KoIonie stimulierender Faktor) vermeiden lassen, wie Heinrich betonte. Eine primär prophylaktische G-CSF-Gabe empfiehlt der Onkologe ab zwei und mehr Vortherapien. Um im Fall einer Diarrhö sofort intervenieren zu können, gibt Heinrich seinen Patienten vorsichtshalber ein Rezept für Loperamid und Ciprofloxacin mit und klärt sie entsprechend auf. Laut Heinrich ist Cabazitaxel nach Docetaxel-Versagen eine wirksame Therapieoption für Patienten mit $\mathrm{mCcRPC}$, die sich mit einem proaktiven Therapiemanagement gut applizieren lässt.

Birgit-Kristin Pohlmann

Satellitensymposium „Moderne Chemotherapie des metastasierten Prostatakarzinoms Anspruch und Realität" im Rahmen des Deutschen Krebskongresses,

Berlin, 24. Februar 2012,

Veranstalter: Sanofi-Aventis, Frankfurt/Main 